\title{
Genetic associations with IBM
}

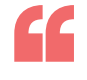

HLA

imputation

identified

three HLA-

$D R B 1$ alleles

that were independently associated with IBM
The HLA region is strongly associated with inclusion body myositis (IBM), according to new findings published in Arthritis \& Rheumatology, confirming an immune-related genetic component in this subtype of myositis. "While a degenerative component is recognised [in IBM], this is the first study to focus solely on immunerelated genes," comments corresponding author Simon Rothwell. "Since there is no proven treatment in IBM, the identification of new genetic risk factors may ultimately lead to new effective treatments."

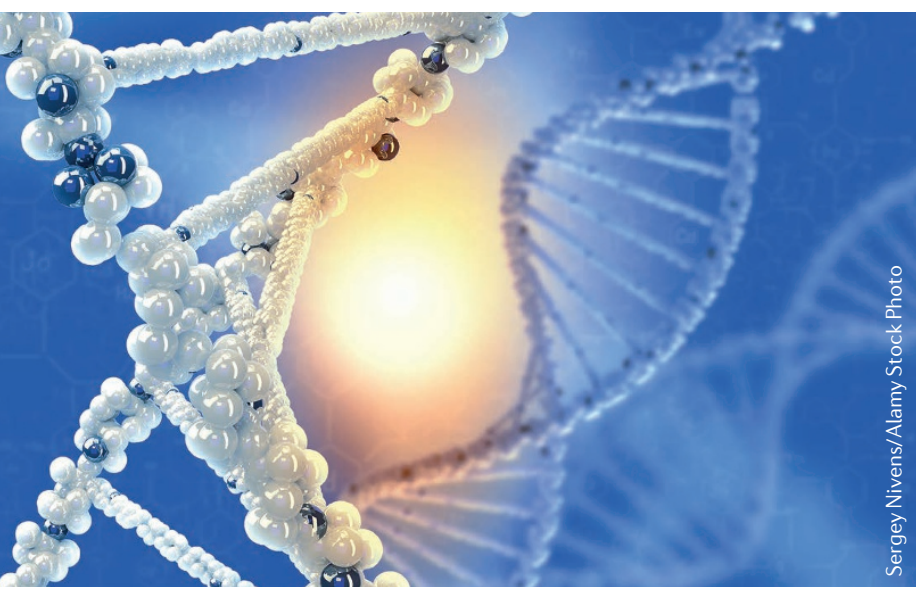

The rarity of IBM has meant genetic studies of this disease have previously been limited. Through the Myositis Genetics Consortium (MYOGEN), Rothwell and colleagues were able to collect samples from 252 patients with IBM, enabling statistically meaningful analysis. The researchers investigated immune-related genes, including those known to be associated with a variety of autoimmune diseases, using the Immunochip singlenucleotide polymorphism (SNP) array. "As well as fine-mapping immune genes, the Immunochip also contains a dense set of SNPs within the $\mathrm{MHC}$ region, allowing for imputation of HLA alleles with high accuracy," explains Rothwell.

In their analysis, SNPs within the HLA region were the only variants to reach genome-wide significance. HLA imputation identified three $H L A-D R B 1$ alleles that were independently associated with IBM: HLA-DRB1*03:01, HLA$D R B 1^{\star} 01: 01$ and $H L A-D R B 1^{\star} 13: 01$. Furthermore, amino acid positions 26 and 11 of $H L A-D R B 1$ were independently associated with disease. "Interestingly, these positions are different than seen in other types of inflammatory myopathy, such as polymyositis and dermatomyositis," remarks Rothwell.

Three non-HLA regions were also suggested to be associated with IBM, including the chromosome 3p21.31 region, which is already known to be associated with various other autoimmune diseases. Within this region, 49 SNPs reached a level suggestive of significance, with SNP rs112088397 showing the strongest association with IBM. Although rs112088397 is located upstream of chemokine receptor 2 (CCR2), further investigation implicated a role for CCR5 in this disease. Going forward, Rothwell explains, they would like to validate the association of CCR5 with IBM, using functional studies to investigate the underpinning disease mechanisms. "This [approach] will assist us in further understanding the pathogenesis of this challenging [and] debilitating disease," he concludes.

\section{Jessica McHugh}

ORIGINAL ARTICLE Rothwell, S. et al. Immune-array analysis in sporadic inclusion body myositis reveals HLA-DRB1 amino acid heterogeneity across the myositis spectrum. Arthritis Rheumatol. http://dx.doi.org/10.1002/ art.40045 (2017) 